\title{
Mangareva, Panthéon de Polynésie sous la direction de Stéphane MARTIN
}

\section{Gilles Bounoure}

\section{OpenEdition}

\section{Journals}

Édition électronique

URL : http://journals.openedition.org/jso/5761

DOI : 10.4000/jso.5761

ISSN : $1760-7256$

Éditeur

Société des océanistes

\section{Édition imprimée}

Date de publication : 30 juin 2009

Pagination : 163-165

ISBN : 978-2-85430-024-6

ISSN : 0300-953x

\section{Référence électronique}

Gilles Bounoure, "Mangareva, Panthéon de Polynésie sous la direction de Stéphane martin », Journal de la Société des Océanistes [En ligne], 128 | janvier-juin 2009, mis en ligne le 30 juin 2009, consulté le 25 septembre 2020. URL : http://journals.openedition.org/jso/5761; DOI : https://doi.org/10.4000/jso. 5761 
deux évasions. Lors de la seconde, ils périrent en mer. Mais loin d'être « la tentation de moribonds pour une dernière aventure magnifique, c'est bien plutôt l'acte d'hommes en rébellion, arrêtés, enfermés, afin de sortir et de dénoncer la réalité carcérale de la lèpre à Rapa Nui, et espérer y mettre un terme » (p. 148). En effet, au fil des années, cette maladie venue de l'extérieur devint un prétexte pour les autorités pour maintenir les Pascuans isolés du monde, puis pour tenir enfermés tous ceux qui furent déclarés lépreux et «tous les rebelles devinrent des lépreux en puissance » (pp. 147148).

Avec la quatrième partie de l'ouvrage, nous suivons l'insurrection de 1964, menée d'une main de maître par Alfonso Rapu, qui aboutit à la promulgation, en 1966, d'une loi qui instaura l'égalité des droits entre les Pascuans et les autres citoyens chiliens.

Dans son dernier chapitre, « Le rire des Pascuans », Marie-Françoise Peteuil nous livre quelques-unes des paroles recueillies au cours de ses entretiens avec les Pascuans de Rapa Nui et d'ailleurs. Ce rire et ses paroles, comme pour lutter contre le silence et l'oubli, montrent les Pascuans, génération après génération, luttant pour prendre en main leur présent et l'avenir de leurs enfants et maintenir la vigueur de leur culture.

Si l'ethnologue Grant McCall avait déjà mentionné en quelques lignes les voyages d'évasion dans son ouvrage de 1980, puis plus longuement en 1997, Marie-Françoise Peteuil nous offre dans Les évadés de l'île de Pâques, la première étude largement documentée, contextualisée et humaine sur cette période, inconnue du public, de l'histoire contemporaine de l'île de Pâques. Un ouvrage essentiel.

\section{RÉFÉRENCES CITÉES}

Guiot Hélène, 2003. Pirogues, reflets de la Polynésie, Paris, Société des Océanistes, Dossier de la Société des Océanistes.

McCall Grant, 1980. Rapa Nui. Traditions and survival in Easter island, Honolulu, University of Hawai'i Press.

—, 1997a. Rapanui Wanderings: Diaspora from Easter Island, Université du Nouveau Mexique.

—, 1997b. Riro, Rapu, Rapa Nui, Rapa Nui Journal 11, 3, pp. 112-122.

PÉTEuIL Marie-Françoise 2003. Ciel d'îles, Journal de la Société des Océanistes 116, pp. 14-24.

Routledge Katherine, 1920 (2e éd.). The Mystery of Easter Island, London, Sifton Praed and Co.

Hélène GuIOT, ArScAn 7041, Nanterre

Martin Stéphane (éd.), 2009. Mangareva, Panthéon de Polynésie, Paris, Somogy-Musée du quai Branly, 80 p., bibliogr., cartes, 46 ill. couleur.

Le musée du quai Branly a eu l'excellente idée de réunir, le temps d'une exposition (3 février-10 mai 2009), les principales sculptures ayant échappé aux autodafés déclenchés par les missionnaires picpuciens à Mangareva, soit une petite dizaine, complétées par une poignée d'objets « ethnographiques » et quelques documents européens. L'ensemble doit ensuite être présenté au musée de Tahiti et des îles (24 juin-24 septembre 2009), à défaut de Mangareva comme il eût été légitime. À Paris, en dépit d'une présentation austère et d'un éclairage parfois insuffisant ou mal réglé, les visiteurs ont eu l'occasion unique de comparer ces pièces venues de musées éloignés ou de collections missionnaires peu accessibles et de les examiner sous leurs principaux angles, grâce à des vitrines judicieusement disposées. Dans sa préface au catalogue de l'exposition, Stéphane Martin parle de « juste retour des choses », à propos des prêts consentis pour cette manifestation par les musées missionnaires légataires d'une histoire accablante. L'expression s'appliquerait tout aussi bien à l'intérêt enfin marqué par le principal musée français d'arts « premiers » à une civilisation et à des insulaires dont ce pays-ci ne s'est guère soucié jusqu'à l'interruption des essais nucléaires dans le Pacifique et à l'examen très récent et toujours en cours de leurs séquelles.

Principalement dévolu aux arts et à la culture d'avant les missionnaires, le catalogue ne dit à peu près rien de la condition des habitants actuels de Mangareva et du reste des Gambier, ce dont les lecteurs peuvent s'informer par ailleurs, on en conviendra. On ne sait si c'est la même considération, ou des raisons d'économie, ou quelque diktat de responsables commerciaux qui ont retenu de munir les principales contributions de ce petit volume de références qui auraient à la fois fondé leurs assertions et permis à un public de plus en plus large d'approfondir ses connaissances. Quoi qu'il en soit, l'introduction de Tara Hiquily (pp. 8-14) et son essai sur « la représentation humaine à Mangareva et dans les autres îles de Polynésie » (pp. 58-69), comme celui de Jean-Marc Pambrun ( Des dieux, des plantes et des constellations », pp. 71-77) ne sont réellement intelligibles ou appréciables que des connaisseurs ayant en tête ou sous les yeux les textes classiques sur Mangareva et pouvant démêler, dans tel paragraphe, ce qui revient à Beechey, à Laval ou à Te Rangi Hiroa, et ce qui relève des hypothèses de leur citateur. L'estimation de la population avancée pour 1825 dans la chronologie (« de 6000 à 8000 habitants », p. 19) ne doit rien à l'escale de Beechey cette année-là ( $\ll 1500 »$, citation exacte p. 57), mais a été formulée pour une période plus ancienne par A. C. Eugène Caillot (1914 : 233), dont la bibliographie du catalogue ne cite aucun des ouvrages, malgré leur importance pour l'histoire de Mangareva. On ne peut croire qu'en préparant leur contribution (« Origine, peuplement et archéologie», pp. 52-57), Éric Conte et Patrick V. Kirch aient songé à minimiser ou à passer sous silence les recherches pionnières de leur collègue, doyen et ami Roger C. Green, qui continue d'étudier ses trouvailles de 1958 à Mangareva et, s'ils y font allusion sans le citer nommément, il faut l'attribuer aux contraintes qui leur ont été imposées. Seules échappent à ce défaut les descriptions des sculptures (pp. 22-44) par Catherine Orliac et, pour l'une d'entre elles, par Laurent Guillaut (d'après les obser- 
vations d'Hélène Guiot), accompagnées des références d'usage. S'il s'agissait de mieux toucher le " grand public », ce catalogue a été conçu ici à rebours du but visé.

Les images qu'il rassemble, les objets parfois inédits qu'il réunit sous un format commode constituent de ce fait son apport le plus précieux. Deux objets supplémentaires (un tambour, un "support à offrandes ») annoncés comme devant venir de la Kunstkammer de Saint-Pétersbourg, mais « non venus » (aléas habituels des expositions) auraient pu être reproduits et décrits, puisque $C$. Orliac les a étudiés dans deux publications que cite le catalogue. Il n'aurait guère été coûteux de signaler les principaux musées de la planète conservant des objets des Gambier, tel le Bernice P. Bishop Museum d'Honolulu (au moins 233 objets, dont des tapas, des colliers, des pagaies, objets absents de l'exposition comme du catalogue du musée du quai Branly). Une illustration au moins aurait dû être écartée ou présentée autrement qu'avec cette légende (p. 15) accumulant les erreurs :

«Guerrier de Mangareva, gravure d'après un dessin de L. Massard réalisé lors du séjour de F.W. Beechey en 1825-1826, in Albert Montemont, Histoires universelles des voyages, 1833, vol. 47 (planches). »

À côté de la liste des officiers du Blossom publiée par Beechey (1831, t. I : vii) qui permet par exemple d'authentifier le dessin de William Smyth également reproduit par le catalogue (p. 13), la Bibliothèque universelle des voyages figure dans la plupart des bibliographies sérieuses, certaines allant jusqu'à mentionner ses principaux illustrateurs. Si Léopold Massard (1813-1889), issu d'une lignée bien connue de dessinateurs-graveurs, ne s'embarqua jamais comme pilotin français à bord d'un navire de guerre britannique partant visiter les sauvages, ses planches attestent un goût hardi de la composition à partir d'éléments disparates qui lui assura un certain succès en son temps. Ses mânes ont dû frémir d'apprendre qu'un de ses dessins avait été pris en 2009 pour une vulgaire esquisse « d'après nature ».

Quelques objets reproduits dans ce catalogue appellent des remarques. Ainsi en est-il (p. 46) de la « tête de lance » en os de cétacé, « collectée entre 1821 et 1824 par George Bennet de la London Missionary Society ». La légende ne le signale malheureusement pas, alors que Steven Hooper, familier de cet objet qui appartint à son grand-père, l'avait antérieurement relevé (2005 : 167). Bennet n'a jamais visité les Gambier - avant ou après Beechey, peut-on ajouter, notamment d'après le récit qu'il a publié en 1831 de sa mission entre 1821 et 1829 dans le Pacifique. Quel a pu être son pourvoyeur? À propos de la « figure de divinité » (LMS 99) que le British Museum tient des collections de la même société missionnaire, C. Orliac (p. 28) suggère qu'elle «a vraisemblablement été collectée par le capitaine Henry, qui faisait du commerce entre Mangareva et Tahiti », ajoutant que ce marin et trafiquant « appartenait à l'une des premières familles de pasteurs de la LMS qui vinrent s'installer à Tahiti en 1797 ». Elle mentionne « l'image » de divinité qu'il avait procurée à Moerenhout et conclut qu'il est « pro- bable que le capitaine Henry collecta, à cette occasion, une seconde idole pour la London Missionary Society ». On ne voit pas que la « tête de lance » venue entre les mains de Bennet probablement à Tahiti, lors de sa tournée d'inspection des postes de la LMs dans le Pacifique, ait pu avoir un autre pourvoyeur que Samuel Henry, dont les exploits précoces sont mis en lumière par Rhys Richards («The Pirates at Tahiti in $1822 »)$ dans le présent JSO.

$\mathrm{Au}$ rapport de Moerenhout (1837, t. I : 95 et II : 326), Henry, que Pomaré avait paré à Tahiti du titre de "Ariki taï (le Chef du dehors, le Chef de la mer)», s'était « lié d'une amitié si étroite avec les chefs des îles Gambier qu'ils ont fait travailler leurs peuples pour lui, et qu'il y est lui-même honoré et obéi, comme s'il y avait de l'autorité ». Établi à son propre compte, son ancien second le capitaine Ebrill y jouissait d'un prestige inférieur mais très suffisant à ses affaires. C'est avec ce dernier que Moerenhout visita Mangareva en février 1834 , notant dans son journal (1837, t. I : 97-98) :

« Les petits autels en bois, placés sur le pavé qui garnissait le devant de la maison, me firent juger que c'était un maraï. Ces autels étaient surmontés de morceaux de corail disposés en corbeille, où se trouvaient du poisson et d'autres comestibles ; et à l'une des extrémités s'élevait une image de trois pieds de haut, assez bien sculptée, et prise dans de justes proportions, à l'exception des bras qui étaient trop minces. Les naturels me dirent que c'était un $t i$, divinité secondaire, placée là pour marquer les limites du lieu sacré. »

Il ajoutait, dans une note rédigée au plus tard en juin 1835 :

« Le capitaine Henri m’a procuré une de ces images, que j'ai en ce moment à Paris. »

S'il fallait probablement alors un Henry pour obtenir ce que ni Ebrill ni Moerenhout n'auraient pu recueillir eux-mêmes à Mangareva, il serait précieux aujourd'hui de connaître le sort de cette "image » arrivée à Paris après avoir été probablement montrée aux États-Unis, où Moerenhout avait réussi à se faire nommer consul, et à se faire prêter 500 dollars d'un négociant de Chicago (Wilks, 1844:65sq). La vendit-il en France ou la rembarqua-t-il dès le même été dans le brick chargé de marchandises prises à crédit qui le ramena à Papeete, où il était également criblé de dettes? Cette note de 1835 ressemble assez à une " petite annonce », sous la plume de ce trafiquant avisé et sans scrupules.

Peut-être doit-on attribuer aux mêmes personnages le parcours initial de la « figure de divinité » masculine redécouverte au musée de Cahors en 2001, parmi les dons venus du capitaine "Bonafous-Murat» (lire Bonnafous-Murat, 1788-1864). Nommé en 1831 au commandement d'un navire lancé en 1828 , la corvette Thisbé (dénomination officielle, et non "Thysbée » comme on lit p. 32), puis de la station du Pacifique dont les vaisseaux parcoururent surtout les côtes chiliennes et péruviennes, cet officier ne visita jamais les Gambier ni même Tahiti, avant son retour à Rochefort-sur-Mer en juillet 1834 et le don qu'il fit au musée départemental du Lot, en 1836 au plus tard (mais non « 1832 », comme mentionné pp. 19 et 32). C'est ce qui conduit le conservateur actuel de ce musée, 
L. Guillaut, à supposer que cet objet a été « probablement reçu sur les côtes du Chili des mains de Jacques Antoine Moerenhout ». Mais lui-même n'aurait pu tenir cette « image » que de Samuel Henry, collecteur de perles et de nacres aux Gambier, et celui-ci n'aurait rien perdu à aller les vendre lui-même à Valparaiso, plutôt que de les confier à un intermédiaire comme Moerenhout. Il n'avait peut-être pas moins d'intérêt que ce dernier à amadouer par un cadeau diplomatique l'officier français le plus gradé alors en poste dans cette région du Pacifique, au moment où les appétits européens commençaient à s'aiguiser à propos de Tahiti. C'est à Henry, actif aux Australes dès 1822 (voir l'article précité de Rhys Richards), plutôt qu'à Moerenhout, qui n'y séjourna que trois fois (1837, t. I : 148), qu'on serait encore tenté d'attribuer le don des trois pagaies de cet archipel que le musée de Cahors tient de Bonnafous-Murat.

Enfin, pour compléter les développements avancés par T. Hiquily sur la figuration humaine en Polynésie, il n'est pas inutile de consulter les éléments publiés par le site électronique du British Museum à propos d'une petite sculpture anthropomorphe $(\mathrm{Oc},+.1997 \mathrm{a})$, haute de $63 \mathrm{~cm}$, jadis reproduite par Terence Barrow comme typique de l'art tahitien (1979 : 20). Les huit photographies mises en ligne sont accompagnées de commentaires de spécialistes débattant de l'attribution de cette statuette, "vraisemblablement des Gambier », estimait en 2003 Véronique Mu-Liepmann, conservatrice du musée de Tahiti et des îles, «typiquement de Tahiti pour le corps », jugeait en 2008 T. Hiquily, non sans ajouter que le visage et les membres rappellent plutôt les Gambier ou les Australes. Les lecteurs, spécialistes ou profanes, trouveront là non seulement un prolongement possible à cette exposition, mais une véritable « leçon de choses » sur les difficultés ou les obscurités entourant encore les arts traditionnels de Polynésie.

\section{RÉFÉRENCES CITÉES}

BArrow Terence, 1979. L'art de Tahiti, Paris, Éditions du Chêne.

BEECHEY Frederick W., 1831, Narrative of a Voyage to the Pacific and Bering's Strait..., London, Colburn \& Bentley.

BRITISH MusEum, http://www.britishmuseum.org/ research/search_the_collection_database.aspx

Caillot A. C. Eugène, 1914. Mythes, légendes et traditions des Polynésiens, Paris, Leroux.

HoOPER Steven, 2005. Pacific Encounters. Art and Divinity in Polynesia 1760-1860, London, The British Museum Press.

Moerenhout Jacques Antoine, 1837. Voyages aux îles du Grand Océan, Paris, Arthus Bertrand.

WILKs Mark, 1844. Tahiti: containing a Review of the Origin, Character, and Progress of French Roman Catholic Efforts for the Destruction of English Protestant Missions in the South Seas, London, John Snow.

Gilles BounOuRE
Al Wardi Sémir, 2008. Tahiti Nui ou les dérives de l'autonomie, Paris, L'Harmattan, 263 p., bibliogr. WitTERSheim Éric, 2006. Après l'indépendance. Le Vanuatu, une démocratie dans le Pacifique, Montreuil, Aux lieux d'être, 190 p., bibliogr., ill., cartes, index.

L'ouvrage de science politique de Sémir Al Wardi intitulé Tahiti Nui ou les dérives de l'autonomie vise à décrire et expliquer les transformations institutionnelles et la vie politique locale en Polynésie française de 1996 à 2006, dans un contexte marqué par la fin des essais nucléaires.

Le premier chapitre revient sur «l'obsession de la France ", c'est-à-dire sur l'importance des relations entre la Polynésie française et la France, dans les débats locaux, illustrée, depuis le début des années 1980, par une bipolarisation de la vie politique locale entre deux grands partis politiques : le Tavini Huiraatira, parti indépendantiste d'Oscar Temaru et le Tahoeraa Huiraatira, parti autonomiste de Gaston Flosse prônant le maintien de la Polynésie française au sein de la république. Pour Flosse, défenseur de l'autonomie, celle-ci vise à octroyer toujours plus de pouvoirs aux institutions de la Polynésie française. Cette compréhension de l'autonomie conduit selon l'auteur à une " surenchère statutaire » dont on peut se demander si elle ne relativise pas la frontière entre une autonomie "hégémonique » et une indépendance accompagnée du maintien des relations avec l'État français.

Le second chapitre revient, dans une perspective historique et juridique, sur la construction d'un pouvoir local. Établissements français d'Océanie de 1880 à 1945, territoire d'outre-mer de 1945 à 2003, la Polynésie française est, depuis 2004, une collectivité d'outremer. Mais différentes revendications autonomistes ne sont pas satisfaites : « les lois de pays » n'ont pas de valeur législative (mais simplement administrative); de même, la "citoyenneté polynésienne" perd son caractère politique. Néanmoins, l'absence de débat sur ce nouveau statut d'autonomie « constitutionnelle » associée à une présidentialisation du régime en Polynésie française a finalement joué en défaveur de l'initiateur de ce nouveau statut, Gaston Flosse, lors de la campagne électorale en avril-mai 2004.

Le chapitre suivant, après une brève réflexion sur l'avenir économique de la Polynésie française, suite à l'arrêt des essais nucléaires, revient sur la «tentation autoritaire » de Gaston Flosse dans un contexte de majorité absolue du Tahoeraa à l'assemblée territoriale. Plusieurs éléments permettent à l'auteur d'étayer ses propos : le fonctionnement peu démocratique du parti politique de Gaston Flosse qui refuse tout pluralisme interne; les pressions exercées sur les associations et les entreprises qui dépendent financièrement des subventions ou des marchés publics ; enfin, le refus du contrôle exercé par l'État et, notamment, par le tribunal administratif. Mais cette « tentation autoritaire » n'aurait pu se réaliser sans la complicité de l'État français qui ne s'est pas donné les moyens d'exercer un contrôle rigoureux sur les décisions prises par les institutions de Polynésie française. 